
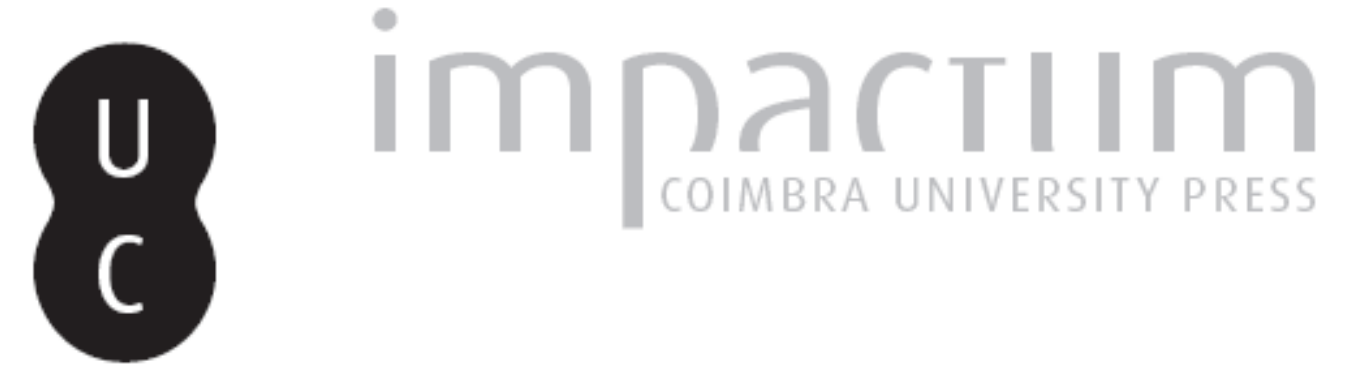

\title{
Pombal et l'enseignement: quelques notes sur la réforme des Estudos Menores
}

Autor(es): $\quad$ Marcadé, Jacques

Publicado por: Imprensa da Universidade de Coimbra

URL persistente:

URI:http://hdl.handle.net/10316.2/43888

DOI:

DOI:https://doi.org/10.14195/2183-8925_4-2_1

Accessed : $\quad$ 26-Apr-2023 03:26:46

A navegação consulta e descarregamento dos títulos inseridos nas Bibliotecas Digitais UC Digitalis, UC Pombalina e UC Impactum, pressupõem a aceitação plena e sem reservas dos Termos e Condições de Uso destas Bibliotecas Digitais, disponíveis em https://digitalis.uc.pt/pt-pt/termos.

Conforme exposto nos referidos Termos e Condições de Uso, o descarregamento de títulos de acesso restrito requer uma licença válida de autorização devendo o utilizador aceder ao(s) documento(s) a partir de um endereço de IP da instituição detentora da supramencionada licença.

Ao utilizador é apenas permitido o descarregamento para uso pessoal, pelo que o emprego do(s) título(s) descarregado(s) para outro fim, designadamente comercial, carece de autorização do respetivo autor ou editor da obra.

Na medida em que todas as obras da UC Digitalis se encontram protegidas pelo Código do Direito de Autor e Direitos Conexos e demais legislação aplicável, toda a cópia, parcial ou total, deste documento, nos casos em que é legalmente admitida, deverá conter ou fazer-se acompanhar por este aviso.

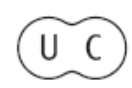




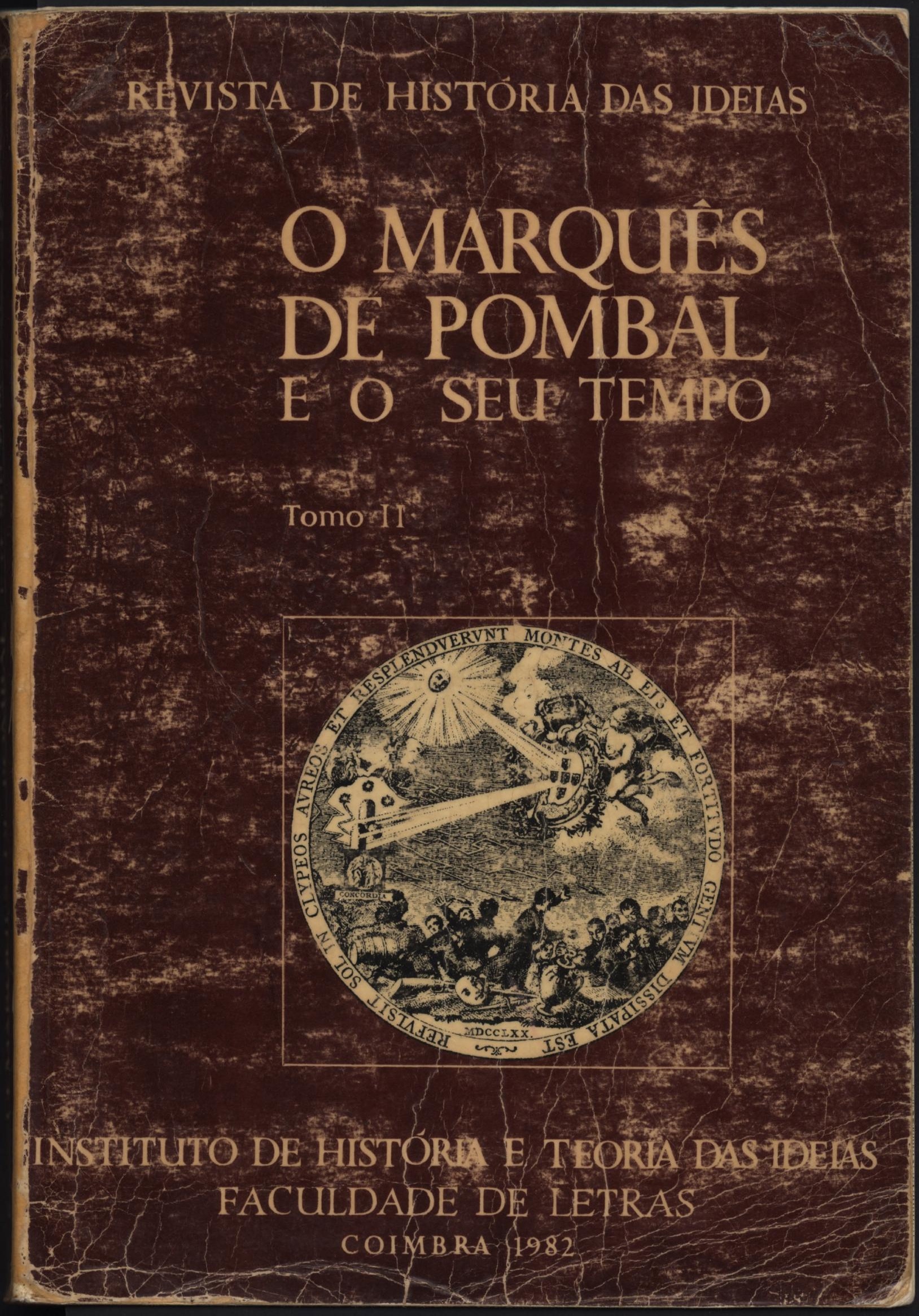




\section{POMBAL ET L'ENSEIGNEMENT: QUELQUES NOTES SUR LA RÉFORME DES ESTUDOS MENORES}

Dans les études générales sur le despotisme éclairé, Sebastião José de Carvalho e Melo ( $\left.{ }^{1}\right)$ est, surtout dans les manuels français, réduit à la portion congrue. Il a pâti du prestige des autres despotes éclairés, prestige bien souvent abusivement suscité par les philosophes du XVIII' siècle, tout particulièrement les français. De son oeuvre, on retient essentiellement la lutte contre les Jésuites et quelques tentatives d'ordre économique. Au plus, dans le prolongement des études sur la politique religieuse et l'expulsion des Jésuites, débouche-t-on sur sa politique scolaire. Mais, bien souvent, le développement se réduit à la seule réforme de l'Université de Coimbra; rares sont les ouvrages qui font allusion à cet autre type $\mathrm{d}^{\prime}$ enseignement que sont les estudos menores $\left({ }^{2}\right)$. Or, c'est tout à l'honneur de Pombal, et de son illustre collaborateur D. Manuel do Cenáculo Vilas Boas, d'avoir tenté de mettre en place, à ce niveau, un enseignement d'Etat. Des établissements d'enseignement ont pu être créés en Prusse, en Autriche... mais c'est au profit des classes moyennes sur lesquelles les régimes voulaient s'appuyer. C'était déjà un enseignement d'un niveau

* Faculdade de Ciências Humanas da Universidade de Poitiers.

(') Comte d'Oeiras, puis marquis de Pombal en 1769. Dans la suite du texte, nous parlerons simplement de Pombal, nom sous lequel il est entré dans l'histoire.

$\left({ }^{2}\right)$ Cela va d'une brève allusion dans l'ouvrage de synthèse de Philippe Sagnac, La fin de l'Ancien régime et la Révolution américaine, col. Peuples et Civilisations, t. XII, $3^{\circ}$ ed. Paris, 1952, à de plus longs développements sous la plume de ces excellents connaisseurs de l'histoire portugaise que sont Albert Alain Bourdon, Histoire du Portugal, Paris, 1970 et Yves Bottineau. Le Portugal et sa vocation maritime, Paris, 1977. 
élevé, qui supposait au préalable une formation primaire. Ces préoccupations ne sont pas étrangères à Pombal: songeons, tout d'abord à l'expérience du Collège des nobles $\left({ }^{3}\right)$, puis à la création des collèges royaux. Mais, lui-même et $\mathrm{D}$. Manuel do Cenáculo ont su mener de pair les deux réformes: celle des études secondaires et celles des études primaires, pour reprendre une terminologie contemporaine $\left.{ }^{4}\right)$. Contre l'opinion du temps, ils ont étendu cet enseignement jusqu'au stade élémentaire, celui de l'apprentissage de la lecture et de l'écriture. En outre, contre toute traditions, ils ont tenté de mettre en place un enseignement d'Etat, ce qu'a souligné António José Saraiva $\left({ }^{5}\right)$. De même que le Portugal, dès la fin du Moyen Age, avait innové en mettant en place un véritable corps de fonctionnaires, de même, au temps des Luzes, il innove en créant un enseignement d'Etat $\left(^{6}\right)$. Cette intervention de l'Etat dans la formation des esprits est tout à fait dans la logique de la politique de Pombal; mais, dans l'Europe du XVIII' siècle, c'est un précédent.

Il n'est pas question, dans ce bref travail, de reprendre l'histoire de l'enseignement au Portugal ( $\left.{ }^{7}\right)$. Nous voudrions simplement tenter de montrer l'originalité de la démarche et les efforts accomplis, en limitant, dans ce cas, les exemples à quelques réalisations dans le sud du pays.

(') Rómulo de Carvalho, História da fundação do colégio real dos Nobres de Lisboa (1761-1772), Coimbra, 1959.

( $\left.{ }^{*}\right)$ L'étude du diocèse de Beja nous incite à nous inscrire à faux contre l'affirmation de Mário Domingues, O marquês de Pombal, o homem e a sua época, Lisboa, $1970, \mathrm{pp}$. 351, 358. Il y a vraiment eu des réalisations, et des résultats.

$\left(^{(5)}\right.$ História da literatura portuguesa, Porto, p. 499.

( ${ }^{\circ}$ Il y a peut-être là une conception de l'Etat - qu'il n'est pas question d'aborder dans cette étude - mais qui, par sa continuité, est exemplaire dans l'Europe d'Ancien régime.

( $)$ Cette étude ne veut point être exhaustive. Il y aurait un livre - et non un article - à écrire, d'après le sous-titre de l'article de António Alberto Banha de Andrade, «A reforma pombalina dos estudos menores em Portugal e no Brasil», Revista de História, (São Paulo), n. ${ }^{\circ} 112,1977$, pp. 459-498. Outre les grandes synthèses de $\mathrm{Da}-$ mião Peres, António Henrique de Oliveira Marques, Joaquim Veríssimo Serrão... nous avons essentiellement utilisé les travaux de: António Ferrão, O marquês de Pombal e as reformas dos estudos menores, Lisboa, 1915 - Maria Adelaide Salvador Marques, A Real mesa censória e a cultura nacional. Aspectos da geografia cultural portuguesa no século XVIII, Coimbra, 1963 - António Cruz, «Nota sobre a Reforma pombalina da instrução publica», Revista da Faculdade de Letras, (Porto), série História, II, 1971, pp. 1-63. 
Pour étudier la politique menée par Pombal dans le domaine de l'enseignement, il faudrait, comme le rappelle fort justement António Alberto Banha de Andrade $\left({ }^{8}\right)$, distinguer deux tranches chronologiques: 1759-1771 et 1771-1777. Dans cette étude, c'est la seconde période qui nous retiendra plus particulièrement. En effet, la première est - le terme ne veut nullement être péjoratif - négative: il s'agit essentiellement de remplacer un système d'enseignement; toute la politique scolaire a été anti-jésuite. La seconde, grâce aux réalisations et au travail déjà accomplis, a pu être créatrice et originale. Et c'est dans ce domaine que réside toute la valeur de la politique menée durant quelques années par Pombal et ses collaborateurs.

Par décret du 6 juillet 1759, D. Tomás de Almeida, neveu du patriarche de Lisbonne $\left({ }^{\circ}\right)$, s'était vu confier la Direction générale des études. C'est à lui qu'a incombé la lourde tâche de mettre en place tout un réseau de collèges royaux destinés à remplacer les anciens établissements jésuites. Rapidement, des commissions adéquates ont fonctionné et le recrutement de maîtres qualifiés a pu être assuré. Dès 1759 , nous trouvons des professeurs royaux de latin et de rhétorique à Lisbonne, de latin à Coimbra et Evora $\left({ }^{10}\right)$. Les choix sont parfois excellents: qu'il nous soit permis de rappeler que, depuis 1764, Bento José de Sousa Farinha était professeur de philosophie à Evora ( $\left.{ }^{11}\right)$. Mais, et l'exemple français le montre bien $\left({ }^{12}\right)$, on ne saurait remplacer en quelques mois, ni même en quelques années, une congrégation qui, depuis plus de deux siècles, avait tissé un réseau d'établissements couvrant tout le pays et modelé des générations entières. Avec pourtant plus de moyens, les réformateurs français avaient en grande partie échoué. De là, sans nul doute, la relative lenteur dans la mise

\footnotetext{
( $\left.{ }^{8}\right)$ A. A. Andrade, art. cit., Revista de História, 1977, p. 459.

(") Dicionário de história da Igreja em Portugal, 1979, 3, pp. 151-152.

${ }^{\left({ }^{10}\right)}$ José Silvestre Ribeiro, História dos estabelecimentos scientificos, literários e artisticos, nos successivos reinados da Monarchia, t. I, p. 211 - A. A. Andrade, art. cit., Revista de História, 1977, p. 471.

(11) A. A. Andrade, ibidem, p. 488 - Maria Amália Machado Santos, José de Sousa Farinha e o ensino, Coimbra, 1948.

${ }^{\left({ }^{2}\right)}$ Jean Morange, Jean-François Chassaing, Le mouvement de reforme de l'enseignement en France, 1760-1798, Paris, 1974, pp. 56-92.
} 
en place du nouveau corps professoral; de là, aussi, bien des hésitations en matière pédagogique, puisque la seule consigne était de prendre le contre-pied de tout ce que faisaient les Jésuites. Le Collège des nobles, au programme trop ambitieux, ne pouvait véritablement servir de modèle et, d'ailleurs, il a rapidement connu un semi échec $\left({ }^{13}\right)$. On comprend que, dans de telles conditions, ce qui a pu être réalisé durant cette période est déjà important $\left({ }^{14}\right)$. Ce travail a été repris et complété durant la seconde phase des réformes, sous la direction de la Real Mesa Censória; et c'est parce que ce travail préliminaire avait été effectué que cette seconde période a pu être constructive.

Il n'est pas question de revenir ici sur les raisons qui ont $\mathrm{pu}$ amener l'éviction de D. Tomás de Almeida $\left({ }^{15}\right)$. En fait, nous semble-t-il, Pombal a surtout voulu confier la responsabilité de la réforme à un homme qui avait déjà fait ses preuves. Par preuves, nous n'entendons pas le préceptorat de l'infant D. José, activité sur laquelle D. Manuel do Cenáculo a toujours été remarquablement discret; mais, nous songeons à la réforme des études dans la Congrégation portugaise du Tiers Ordre régulier de Saint François. Cette réforme, fort bien planifiée tant pour la progression des études que pour la répartition géographique des enseignements, était un modèle d'efficacité $\left({ }^{16}\right)$. D'ailleurs, nombre de congrégations, portugaises comme les Bénédictins ou les Carmes Déchaux, ou étrangères comme la province d'Andalousie des Réguliers du Tiers Ordre franciscain l'on copiée $\left({ }^{17}\right)$. Avec D. Manuel do Cenáculo, Pombal avait désormais, non pas une créature - nous nous sommes élevé contre cette interprétation $\left({ }^{18}\right)$ - mais un praticien compétent qui s'avérait un collaborateur dévoué. Dans un siècle où l'expérience devient reine, c'est, à notre avis, une des raisons qui ont fait préférer D. Manuel do Cenáculo

(13) R. de Carvalho, op. cit., p. 146 et seq.

(') A. A. Andrade, art. cit., Revista de História, 1977, p. 461.

( $\left.{ }^{15}\right)$ Idem, ibidem, p. 490.

(10) Jacques Marcadé, «D. Fr. Manuel do Cenáculo Vilas Boas, Provincial des Réguliers du Tiers ordre franciscain 1768-1777», Arquivos do Centro Cultural Português, vol. III, pp. 431-458.

${ }_{\left({ }^{17}\right)}$ A. A. Andrade, "A orientação do estudo de filosofia nos Franciscanos (século XVIII), Brotéria, 1946, p. 35 - Trigoso d'Aragão Morato, «Elogio histórico... D. Frei Manoel do Cenáculo...», História e Memórias da Academia Real das Sciencias de Lisboa, IV, I, p. LXXIX. En 1776, ce plan fut même étendu à toutes les familles franciscaines de la péninsule ibérique.

( $\left.{ }^{18}\right)$ D. Manuel do Cenáculo Vilas Boas, évêque de Beja, anchevêque d'Evora 1777-1814, Paris, 1978, pp. 55, 59, 84, 480-481. 
à D. Tomás de Almeida, bien que ce dernier n'ait nullement démérité; de même, $\mathrm{D}$. Manuel do Cenáculo avait été, pour cette même raison, préféré à António Pereira de Figueiredo, en tant que précepteur du prince héritier.

La Direction générale des études était supprimée en tant qu'organisme autonome et la gestion des écoles fut confiée à la Real Mesa Censória, présidée depuis le 16 mars 1770 par D. Manuel do Cenáculo. L'alvará du 4 juin 1771 mit fin à la mission de D. Tomás de Almeida qui, le $1^{\text {er }}$ juillet 1771 dut remettre tous les papiers de son administration à la Real Mesa Censória. C'est à cette dernière, et surtout à son président, qu'incombait désormais la charge, non seulement de gérer le réseau déjà en place mais aussi de créer de nouveaux établissements.

En effet, même au niveau des écoles élémentaires, la Real Mesa Censória n'est pas partie de zéro et la mise en place des nouveaux établissements n'est pas une création ex nihilo. Des écoles de village existaient dans certaines paroisses d'Algarve, comme en témoignent les préoccupations de D. Ignacio de Santa Thereza, dans ses pastorales $\left({ }^{19}\right)$. Elles sont fort nombreuses dans le nord du Portugal, comme le souligne Domingos Vandelli dans un de ses mémoires $\left({ }^{20}\right)$; et si, comme il l'indique, elles sont peu nombreuses dans le sud, elles ne sont pas pour autant inexistantes. Nous apprenons, au hasard des sources, l'existence de certaines de ces écoles: ainsi, à São Miguel do Pinheiro, avec le vicaire du lieu $\left({ }^{21}\right)$, ou bien à Serpa, et dès 1771, l'instituteur du lieu, Gaspar Honorato da Mota e Silva en profite pour poser sa candidature à un futur poste d'Etat $\left({ }^{22}\right)$. Dans la première période, $D$. Tomás de Almeida avait publié des instructions pour les mestres de ler e escrever. Elles sont très significatives de cette période et, en particulier, elles montrent l'absence de rupture entre gouvernement et Eglise, du moins tant que celle-ci se cantonne dans un rôle religieux et ne prétend pas intervenir sur un plan politique. Les instituteurs devaient enseigner la lecture, l'écriture, en gros caractères, les quatre opérations, mais aussi le catéchisme. Ces instructions ont été maintenues par la suite, car c'est

${ }^{\left({ }^{10}\right)}$ René Renou, L'Algarve au XVIII ${ }^{\circ}$ siècle, thèse de $3^{\circ}$ cycle, Paris X, p. 242.

$\left.{ }^{20}\right)$ Biblioteca da Manisola (Evora), n. ${ }^{\circ} 486$. Texte sans titre, sur l'agriculture portugaise.

(21) Arquivo distrital de Evora (A.D.E., par la suite) $\mathrm{CXXX} / 2-$ -16. Il recevait $1 / 2$ alqueire par feu (16,56 litres, mesure de Mértola) soit, en 1770 l'équivalent de 15000 réis.

${ }^{(22)}$ A.D.E. CXXVII/1-10, n 1880 , du 15 août 1771. 
ce que D. Manuel do Cenáculo semble attendre des instituteurs qui se trouvent dans son diocèse $\left({ }^{23}\right)$. A ces écoles, privées pourrait-on dire, la Real Mesa Censória a voulu ajouter 440 postes de mestres de ler royaux $\left({ }^{24}\right)$.

Et, ce projet du 12 novembre 1772 n'est pas resté un voeu pieux, car l'Etat s'est donné les moyens nécessaires pour mettre en place cet enseignement. Sur ce point, nous pouvons noter la très grande différence entre l'expérience portugaise et celle que connaissent d'autres pays. En Angleterre, pays le plus alphabétisé à cette époque, l'enseignement est entièrement libre; l'Etat n'intervient nullement $\left({ }^{25}\right)$. En France, depuis la Déclaration royale de 1724 , les paroisses étaient tenues de créer des écoles; mais, l'intervention de l'Etat s'est limitée à cette décision $\left({ }^{26}\right)$. Même en Pologne, où la Commission d'Education a certes accompli une grande oeuvre, les écoles primaires ont été négligées et la Commission s'en est remise à la bonne volonté des curés et des propriétaires $\left({ }^{27}\right)$. Dans l'empire, malgré les réticences de certains à l'encontre de l'enseignement primaire, une commission dirigée par J-Ig Felbiger, abbé de Sagan, s'y est intéressée; les réalisations, d'ailleurs à une date plus tardive qu'au Portugal, ne semblent pas avoir dépassé le stade des projets $\left({ }^{28}\right)$. Au Portugal, par contre, pour créer un enseignement d'Etat, des crédits d'Etat ont été trouvés. C'est d'ailleurs ce qui justifie le contrôle sur le recrutement, alors que, en France, il suffisait d'une autorisation accordée par les autorités ecclésiastiques pour pouvoir être recruté comme maître d'école par une communauté d'habitants. Le paiement d'un salaire justifie pleinement la surveillance officielle et aussi, par ailleurs, a peut-être facilité le recrutement.

A cet effet, a été crée un nouvel impôt, le subsidio literário. D. Manuel do Cenáculo, déjà président de la Real Mesa Censória, ayant été nommé le 10 novembre 1772 président de

${ }^{(2)}$ A.D.E. CXXVIII/2-4, Lettre pastorale du 8 septembre 1777.

${ }^{(24)}$ Nous n'étudions ici que la métropole et ne tenons pas compte, de ce fait des créations pour les îles, l'Afrique, l'Asie et le Brésil.

$\left.{ }^{25}\right)$ Ivy Pinchbeck et Margaret Hewitt, Children in english society, Londres, 1969 - Jonh Lawson \& Harold Silver, A social History of education in England, Londres, 1973.

${ }^{\left({ }^{2}\right)}$ Ainsi, deux paroisses proches de Poitiers, capitale régionale et ville épiscopale, n'ont pas eu d'instituteurs, ou alors épisodiquement, au XVIII' siècle; le taux d'alphabétisation est inférieur à $10 \%$.

${ }^{\left({ }^{27}\right)}$ Ambroise Jobert, La Commission d'Education nationale en Pologne (1773-1794), Paris, 1941, p. 275. p. 221.

${ }^{23}$ ) V-L Tapié, Monarchies et peuples du Danube, Paris 1969, 
la Junta do subsidio literário, il avait ainsi la haute main et sur l'enseignement et sur son financement. Certes, les multiples charges qu'il assumait l'ont amené à déléguer, dans le cas du subsídio literário une partie des responsabilités à Fr. Joaquim de Sant'Ana e Silva; mais, les deux hommes, les échanges de correspondance en témoignent $\left({ }^{28}\right)$, travaillaient en plein accord. La nouvelle charge de l'Etat était donc couverte par un impôt indirect: un real par canada de vin et 4 réis par canada d'eau-de-vie vendues, 160 réis pour chaque pipe d'eau-de-vie fabriquée au Portugal et dans les îles de l'Atlantique $\left({ }^{30}\right)$. Ainsi, en 1776, la comarca d'Evora a fourni plus de 1600000 réis, de 47623 dans le district le plus pauvre, Mourão, à 705216 pour celúi d'Evora $\left({ }^{31}\right)$. Les salaires ne semblent pas très élevés. Pour les mestres de ler, ils seraient de l'ordre de 40 à 60000 réis, soit un peu plus que celui d'un ouvrier, si nous en jugeons par des témoignages contemporains $\left({ }^{32}\right)$. Dans une vila, un professeur de grammaire latine aurait touché 100000 réis ( $\left.{ }^{33}\right)$. Apparemment, les premiers professeurs royaux, nommés dans des villes plus importantes, avaient des salaires plus élevés: 300000 réis à Lisbonne et 240000 pour les enseignants de latin à Porto et Evora ( $\left.{ }^{84}\right)$. Quoiqu'il en soit, la mise en place de tels moyens traduit bien la volonté politique du gouvernement et son désir d'aboutir.

Il n'en avait que plus de mérites, surtout en mettant l'accent sur l'enseignement primaire: $59,2 \%$ des postes créés, si nous songeons à la situation en Europe à cette époque. Pour apprécier à sa juste valeur la tentative de Pombal et les réalisations de la Real Mesa Censória, il nous faut rappeler ce qu'était l'opinion au XVIII ${ }^{e}$ siècle, cette opinion en grande partie façonnée par les philosophes. Un enseignement populaire paraissait un gaspillage inutile, voire dangereux, aux yeux

(") A.D.E. CXXVII/2-13, no 2493 à 2505.

$\left({ }^{20}\right)$ Simão José da Luz Soriano, História da guerra civil e do estabelecimento do governo parlamentar em Portugal, Lisboa, t. II, p. 59.

(") A.D.E. CLXIV/2-33.

(22) Comartin-Desoteux, Voyage du ci-devant duc de Chatelet au Portugal... Paris, an VI, t. II, p. 46. Il estime le salaire journalier à 10-12 sols, le sol valant 10 réis. Compte tenu des jours féries, 30000 réis semble un maximum. Notons, à titre de comparaison, que les desservants des paroisses du diocèse de Beja où ont été implantées des écoles gagnaient de 190000 réis à Mértola jusqu'à 250000 à Moura et même 400000 à Beja.

(æ) A. Ferrão, ob. cit., p. 105 qui, pour les salaires, cite Francisco José dos Santos Marrocos.

(') J. S. Ribeiro, ob. cit., I, p. 211 - A. A. Andrade, art. cit. Revista de História, 1977, p. 471. 
de ces maîtres de la mode et des réputations. Ainsi, Voltaire félicitait La Chalotais qui déclarait qu'il y avait trop d'écoles... A leurs yeux, l'instruction primaire devenait suspecte: "Les Frères des écoles chrétiennes, qu'on appelle Ignorantins, sont survenus pour achever de tout perdre. Ils apprennent à lire et à écrire à des gens qui eussent dû n'apprendre qu'à dessiner et à manier le rabot et la lime» $\left.{ }^{35}\right)$. Louis René Caradeuc de la Chalotais, qui, par ailleurs, prétendait offrir un plan destiné à remplacer l'enseignement des collèges Jésuites, écrivait «Le bien de la société demande que les connaissances du peuple ne s'étendent pas plus que ses occupations" $\left({ }^{38}\right)$. Des économistes, tels Guyton de Morveau ou Mirabeau partageaient ce préjugé et, dans le royaume voisin, leur disciple, Pablo de Olavide, ne se préoccupe guère de l'enseignement primaire ${ }^{{ }^{37}}$ ). De tous les philosophes français, un seul semble avoir-manifesté un intérêt pour ce type d'enseignement. Il s'agit de Denis Diderot, pour, il est vrai, l'empire russe $\left({ }^{38}\right)$. S'il avait connu les réalisations portugaises, on peut supposer que, à la différence des autres philosophes parisiens, il aurait été à même de les apprécier. Mais, heureusement ce n'est pas cette influence qu'a subie le gouvernement portugais. Bien au contraire, dans la ligne des Luzes méditerranéennes, si remarquablement analysées par Luís Cabral de Moncada ( $\left.{ }^{89}\right)$, il a cherché à mettre en pratique les conseils donnés par Vernei: il n'y a pas de prospérité sans un bon système d'éducation $\left({ }^{40}\right)$ et nous ajouterons, sans un système complet. De là, cet intérêt porté à la base même du système, c'est-à-dire l'enseignement élémentaire .

1972 , p. 825.

( ${ }^{(35)}$ Cité in Philippe Ariès, La France et les Français, Paris,

$\left.{ }^{30}\right)$ Essai d'éducation nationale ou plan d'études pour la jeunesse, ed. 1763, pp. 25-26.

${ }^{(37)}$ Marcelin Defourneaux, Pablo de Olavide ou l'afrancesado, Paris, 1959, p. 118.

${ }_{\left({ }^{35}\right)}$ Les plans et Statuts des différents établissements ordonnés par l'Impératrice Catherine II pour l'éducation de la jeunesse, ouvrage dans laquel il demandait que l'enseignement soit ouvert à tous, même les derniers des paysans, ont été rédigés par Diderot au retour de son voyage en Russie et imprimés à Amsterdam en 1775. Cette brochure était connue de $\mathrm{D}$. Manuel do Cenáculo qui y fait allusion dans une circulaire adressée aux enseignants de Beja (Biblioteca da Academia das Ciências, B.A.C. par la suite) 122 V, Aos professores...)

Coimbra, 1950.

(20) Século XVIII - Iluminismo católico-Vernei e Muratori,

( $\left.{ }^{\circ}\right)$ Robert Ricard, «De Campomanes à Jovellanos. Les courants d'idées dans l'Espagne au XVIII siècle, à propos d'un ouvrage récent», Les lettres romanes XI, 1957, pp. 31-52. 
La Real Mesa Censória avait prévu, pour la métrople, la création de 440 postes de mestres de ler et les avait répartis suivant les régions. Il n'est pas question de faire dans cette étude une analyse précise, en tenant compte des chiffres de créations de postes par comarcas ainsi que de la population et de la superficie de celles-ci. Nous voudrions simplement évoquer quelques ordres de grandeur pour essayer de comprendre le travail de la Real Mesa Censória; aussi, plus que les chiffres par comarcas du document officiel $\left.{ }^{(11}\right)$, nous avons préféré prendre les chiffres par provinces et, pour la population, nous avons retenu les estimations faites par Dumouriez $\left({ }^{42}\right)$. Outre le fait que la population est donnée en habitants, ce qui élimine les risques d'erreurs d'une évaluation par feux, nous avons supposé que de tels chiffres étaient acceptés par les autorités administratives qui les avaient communiqués à leur visiteur. Le tableau comparatif que nous pouvons tirer des divers renseignements fournis est intéressant:

\begin{tabular}{|c|c|c|c|}
\hline & & mestres de ler & rapport \\
\hline & & \multirow{2}{*}{$\begin{array}{l}106 \\
131\end{array}$} & \multirow{2}{*}{$\begin{array}{l}1 \text { pour } 6250 \\
1 \text { pour } 4300\end{array}$} \\
\hline & & & \\
\hline & & \multirow{4}{*}{$\begin{array}{l}76 \\
66 \\
51 \\
10\end{array}$} & \multirow{4}{*}{$\begin{array}{l}1 \text { pour } 6650 \\
1 \text { pour } 4200 \\
1 \text { pour } 3100 \\
1 \text { pour } 6500\end{array}$} \\
\hline & & & \\
\hline & & & \\
\hline & & & \\
\hline $\begin{array}{l}\text { Provinces } \\
\text { Estremadura } \\
\text { Beira } \\
\text { Entre Minho e } \\
\text { Douro } \\
\text { Alentejo } \\
\text { Trás-os-Montes } \\
\text { Algarve }\end{array}$ & $\begin{array}{l}\text { population } \\
660000 \mathrm{~h} . \\
560000 \mathrm{~h} . \\
504000 \mathrm{~h} . \\
280000 \mathrm{~h} . \\
156000 \mathrm{~h} . \\
65000 \mathrm{~h} .\end{array}$ & 440 & 1 pour 5000 \\
\hline
\end{tabular}

Nous voyons, en effet, que la Real Mesa Censória ne s'est pas contentée de créations proportionnelles au nombre d'habitants. La Beira, moins peuplée que l'Estremadura a reçu plus de mestres de ler que cette dernière. L'analyse doit être poussée plus loin. Le chiffre de population ne signifie rien en lui-même; si l'on veut que la réforme porte ses fruits, il faut qu'elle tienne compte aussi de l'habitat et des moyens de communication. Il est évident que le secteur touché par une école ne

(11) Biblioteca Nacional de Lisboa (B.N.L. par la suite), Manuscritos, Collecção Pombalina, 455, Colleç̧ão das Leis, 3. Les chiffres par comarcas son reproduits par A. Ferrão, ob. cit., pp. 94-95.

('2) Etat présent du royaume de Portugal en l'année 1766, Lausanne, 1775, p. 56. En 1776, pour 633432 feux, le Portugal aurait eu environ 2500000 habitants, ce qui renforce la crédibilité des chiffres avancés par Dumouriez. Toutefois, pour l'Algarve, R. Renou (ob. cit., p. 44) estime la population, à cette époque, entre 80 et 90000 habitants. 
sera pas le même dans une zone de plaine et dans une zone de montagnes. C'est peut-être ce qui explique le rapport favorable à la Beira. Dans l'Estremadura, près du tiers de la poputation est concentré dans la capitale; dans l'autre province, les districts montagneux l'emportent sur les plaines de la Beira Litoral. En apparences, le Minho serait défavorisé; mais, n'oublions pas que cette zone de vieux peuplement est celle des petits villages densément peuplés. Les 6550 habitants correspondant à une école sont répartis sur combien de kilomètres carrés? Le Trás-os-Montes paraît avantagé avec une école pour 3100 habitants; mais une telle école est sensée desservir $230 \mathrm{Km} 2$, et dans une zone montagneuse. Aussi, dans cette première répartition de novembre 1772, nous sommes tenté de voir le résultat d'une réflexion géographique qui s'est efforcée de prendre en compte tous les paramètres. Il nous faut toutefois noter que rien n'explique le faible nombre de postes affectés à l'Algarve, ni la population, ni la superficie.

Nous voudrions reprendre notre raisonnement en l'appliquant plus concrètement à deux exemples géographiques; le diocèse de Beja qui correspond à deux comarcas et la province d'Algarve. Nous ne reprendrons pas exactement la liste dressée officiellement dans la mesure où Ferreira do Alentejo y figure dans la comarca d'Ourique, alors que João Baptista de Castro et António de Oliveira Freire situent cette vila dans celle de Beja comme le font, par ailleurs, toutes les statistiques du diocèse nouvellement crée ${ }^{\left({ }^{48}\right)}$. Arbitrairement, nous avons rattaché à la comarca d'Ourique le termo d'Odemira, enclave de Beja, mais que l'économie et la géographie rattachent à Ourique et laissé en dehors de la comarca de Beja le termo d'Alcoutim, qui traditionnellement relève de l'Algarve. Nous aurions 8 mestres de ler pour les quelques 55000 habitants de la partie ainsi définie de la comarca de Beja, soit 1 pour 6900 habitants et 9 pour les 45000 de celle d'Ourique, soit 1 pour 5000 . Mais, nous devons corriger ce mode de calcul par celui des superficies. Tous les postes attribués pour Beja, l'ont été pour la partie nord-est, soit la zone la plus riche, avec certains cantons qui, au dire des contemporains, sont les plus fertiles de l'Alentejo. En tenant compte des seules surfaces, les rapports sont inversés: il y a un mestre de ler

(') Mappa de Portugal, Lisboa, $2^{\circ}$ ed., t. 1, p. 76 et, du même, le brouillon conservé à Evora (CXII d/1-12, f. 36) - Descripsam corografia do Reyno de Portugal, Lisboa, 1734, ouvrage de base pour la géographie de la région, d'après Pombal lui-même. 
pour environ $560 \mathrm{Km} 2$ dans la zone riche des gros villages agricoles et un pour près de $800 \mathrm{Km} 2$ dans le sud plus pauvre. Parler en terme de géographie est peut-être un anachronisme et ce serait vouloir prêter aux gens du XVIII ${ }^{e}$ siècle un état d'esprit qu'ils n'avaient point. En fait, nous sommes certes dans une période pré-statistique, où les chiffres précis sont rares; mais il ne faut pas oublier que les gens de la Real Mesa. Censória pouvaient avoir une connaissance solide de leur pays, par les ouvrages, par les résultats d'enquêtes - la localisation des paroisses et les distances sont évoquées dans le Dicionário geográfico - ainsi que, et nous songeons tout particulièrement à $\mathbf{D}$. Manuel do Cenáculo, par leurs voyages. Aussi, de telles préoccupations ont fort bien pu ne pas leur être étrangères.

Dans la comarca de Beja, les postes ont été attribués au chef-lieu et aux deux grosses agglomérations de Moura et Serpa, à raison de 2 chacune; le septième a été implanté à Alvito et nous y ajoutons Ferreira. Dans la comarca d'Ourique, Almodôvar, Mértola et Ourique ont reçu deux postes; Messejana, Santiago do Cacém et Sines, un. Ainsi, pour population inférieure à celle du canton de Moura, Mértola a reçu 2 mestres de ler... mais la superficie est pratiquement du double. Notons, toutefois, une grave lacune. Les deux écoles le plus au sud sont implantées à Mértola et Almodôvar; rien n'a été prévu pour la zonne montagneuse séparant l'Alentejo de l'Algarve. Aucune création sur le versant méridional ne venait pallier cette situation. En effet, les postes envisagés en 1772 sont tous dans des agglomérations de la côte ou son voisinage immédiat, c'est-à-dire la partie la plus riche et la plus peuplée. Castro Marim, Faro, Tavira, se voyaient attribuer deux postes; Alvor, Loulé, Portimão et Silves, un. Dès novembre 1773, la Real Mesa Censória s'était efforcée de combler certaines lasunes et de corriger certains oublis. Quatre nouveaux postes ont été attribuées pour l'extrême sud du pays ( ${ }^{(4)}$ ). Pour l'Algarve, deux ont été affectés à Monchique et Aljezur, ce qui permettait de desservir le nord-ouest montagneux de la province; quant à celui d'Albufeira, il comblait le vide, relatif, entre Portimão et Faro. Dans le cas de Beja, le poste a été attribué à Vidiguei$\mathrm{ra}$, au coeur de la riche et dense région viticole du nord de Beja. Notons que la comarca d'Ourique n'a rien reçu et que le flanc nord de la serra est toujours aussi défavorisé; il fau-

(') B.N.L., Col. Pombalina, 455, col. das Leis, 3. 
dra attendre quelques années pour qu'une initiative privée vienne combler cette lacune $\left(^{45}\right)$.

Parallèlement ont été crées des chaires de grammaire latine: 205, de rhétorique: 39 et de philosophie: 28 , ainsi qu'un enseignement de grec. Pour la répartition géographique, nous renvoyons aux tableaux très complets de l'ouvrage de Maria Adelaide Salvador Marques; ce que nous voudrions souligner ici c'est la progression, la hiérarchie des études. Les chaires de latin représentent à peu près la moitié du nombre de postes d'instituteurs et, au sommet de la hiérarchie, nous trouvons un nombre plus réduit de chaires de rhétorique et de philosophie. Il semblerait que les auteurs du plan aient tenu compte non seulement de la difficulté de recruter des maîtres à un niveau plus élevé, mais aussi de l'effritement logique des effectifs, au fur et à mesure de la progression. Ajoutons, dans ce cursus une petite touche de fantaisie: l'enseignement du grec, alors que ce genre d'études était en totale décadence, non seulement au Portugal, mais dans l'Europe entière $\left(^{46}\right)$. Sans nul doute, était-ce à l'initiative de $\mathrm{D}$. Manuel do Cenáculo, qui sur l'utilité de cette langue, partageait l'intérêt porté par le grand réformateur du royaume voisin, Pedro Rodriguez, comte de Campomanes. Mais, au Portugal, on ne se contentait pas de relancer les études dans les collèges universitaires; des postes de professeur sont prévus, mais difficilement pourvus, dans tous les chefs-lieux de comarcas. Si nous reprenons le cas de Beja, les professeurs royaux se répartissent ainsi: tout en bas de l'échelle 8 (puis 9) mestres de ler, 4 professeurs de grammaire latine: Alvito, Beja, Moura et Serpa (puis, un cinquième à Vidigueira) et, au chef-lieu un professeur de grec, le propre frère d'un des collaborateurs de $D$. Manuel do Cenáculo, un de rhétorique et un de philosophie. Nous trouvons une pyramide semblable, dans l'Algarve encore que la province, avec une superficie et une population supérieures à celle de la comarca de Beja semble un peu défavorisée par rapport à cette dernière. Aux 10 (puis 13) mestres de ler s'ajoutent 4 professeurs de latin et 2 de rhétorique et 1 de philosophie.

Que cette initiative de mise en place d'un enseignement officiel réponde aux voeux des populations, la correspondance reçue par D. Manuel do Cenáculo en témoigne. Villes du Bré-

(45) Sur l'initiative de D. Manuel do Cenáculo qui, au cours d'une visite pastorale, a pris conscience de l'isolement et du retard des populations. Une des deux écoles fondées se trouve à Odemira.

${ }^{\left({ }^{*}\right)}$ Pour le tableau et, surtout, les références, nous nous permettons de renvoyer à notre travail, $D$. Manuel do Cenáculo Vilas Boas..., pp. 17-18. 
sil comme de métropole réclament de hâter les affectactions, ainsi que des créations nouvelles. Par exemple, dans la comarca de Beja, mais aussi le diocèse du Président de la Real Mesa Censória, la municipalité de Vila de Frades réclame à son tour une école $\left({ }^{47}\right)$. Pourtant, malgré les besoins (et les rapports école-superficie ou école-chiffres de population sont assez explicites), la mise en place de l'enseignement d'Etat a été lente et difficile. Il fallait tout d'abord recruter des maîtres qualifiés. Ce fut la tâche de commissions comme celles que mit en place D. Francisco de Lemos, évêque de Coimbra, mais aussi Recteur de l'Université. Ainsi, celle qui, en 1773, était chargée d'examiner les futurs mestres de ler était présidée par Tomás Pedro da Rocha $\left({ }^{48}\right)$. Les examinateurs envoyaient ensuite leur rapport à la Real Mesa Censória, qui tranchait en dernier ressort $\left({ }^{49}\right)$. Ce système n'exclut pas les recommandations individuelles; António dos Santos Simões Boralho, professeur à Evora, avait recommandé un candidat pour le poste de grec à Portalegre $\left({ }^{50}\right)$. L'installation de professeurs a été plus ou moins rapide: en 1774, Tabuaço, dans la comarcu de Viseu, n'avait toujours pas de professeur de grammaire latine; et deux ans plus tard il faudra rapidement trouver un remplaçant, le titulaire, ayant une plume un peu trop acérée, s'était retrouvé en prison $\left.{ }^{51}\right)$. Les conditions de travail n'étaient pas toujours faciles. Ainsi, à Evora, où l'influence des Jésuites était restée forte, António dos Santos Simões Boralho et son collègue Roberto Limpo de Lacerda en font l'expérience, au point même que le second demande son changement $\left.{ }^{52}\right)$.

Enfin, le financement envisagé n'a pas toujours été assuré. Le subsídio literário, nouvel impôt destiné à l'enseignement $\left({ }^{53}\right)$, venait s'ajouter à de multiples autres charges, en particulier la décima devenue définitive à compter du 26 sep-

( $\left.{ }^{47}\right)$ A.D.E. CXXVII/1-10, n० 1817, du 6 janvier 1774.

(48) A.D.E. CXXVII/1-5, n० 776, du 27 mai 1773 et $n^{\circ} 778$, du 27 mai de l'année suivante, où il accuse réception de nouvelles instructions.

$\left.{ }^{(9}\right)$ A.D.E., CXXVII/1-1, no 95, du 17 juillet 1773, de Fr. António Alvares da Silva, qui opérait dans la région de Porto.

${ }^{\left({ }^{5}\right)}$ A.D.E., CXXVIII/1-3, $n^{\circ} 403$, du 15 avril 1775.

(v) A.D.E. CXXVII/2-13, no 4513 du 21 novembre 1774 et CXXVII/1-2, n० 223 du 7 octobre 1776.

(22) A.D.E. CXXVII/1-3, no 402, du 21 novembre 1774 et CXXVII/2-14, du 4 novembre 1775 .

${ }^{\left({ }^{53}\right)}$ Ce n'était pas le seul. Ainsi. la comarca d'Ourique devait verser 73590 réis, en 1774, pour la Faculté de médecine de Coimbra, celle de Beja 180020 et celle d'Evora 405920 (B.N.L., col. Pombalina 455 , col. das Leis 3 ). 
tembre 1762. Or, comme le constatait Juan Alvarez de Colmenar «les impôts sont fort grands au Portugal et assurément on ne pourrait les pousser plus loin sans accabler le peuple» $\left({ }^{54}\right)$. Même si, en apparences, la taxe n'est pas très élevée, 1e gouvernement éprouve bien des difficultés pour la faire rentrer. Il y a mauvaise volonté de certains, tel le collège $\mathrm{N}^{\mathbf{a}} \mathrm{S}^{\mathbf{a}}$ da Estrela de Coimbra, qui prétendait être exempté sous prétexte que le vin qu'il consommait lui avait été offert $\left({ }^{55}\right)$. Très vite les chiffres des rentrées ont diminué et les retards se sont accumulés. La comarca de Viseu a versé 5394998 réis en 1773; le total tombe à 3361158 , en 1774 . Pour celle de Portalegre, les levées sont de 459514 réis, en 1773, 266526 en 1774; pour Santarém, les chiffres sont respectivement de 5413300 et $\left.3513024{ }^{(58}\right)$. En 1776, la comarca d'Evora a payé 1616880 réis et, en 1777, 1092634 seulement $\left({ }^{57}\right)$. En 1775, la comarca de Lamego verse un reliquat de 151000 réis, dûs pour 1772; toutes les taxes dûes pour 1774 n'étaient pas encore perçues l'année suivante pour la comarca d'Aveiro, et même celles de 1773 , dans le cas de l'Algarve $\left({ }^{58}\right)$. De fait, cette initiative généreuse de la part du gouvernement supposait un autre contexte socio économique. On pourrait inverser le jugement de Pierre Chaunu: «il n'est de richesse que d'hommes, mais d'hommes instruits» $\left.{ }^{(89}\right)$. En effet, pour qu'il y ait des possibilités d'alphabétisation et d'instruction, il faut au départ des hommes riches. La carte de l'alphabétisation dans la France du XVIII ${ }^{e}$ siècle, avec le contraste entre la France du nord, riche et instruite, et la France du midi, pauvre et souvent analphabète, le montre bien. Il ne suffit pas de canaliser les bonnes volontés, d'imposer une politique; encore fallait-il en avoir les moyens.

Nous ne disposons guère de jugements sur les professeurs royaux. Les deux seuls que nous avons pu consulter sont, par leur nature même, quelque peu sujets à caution. Le premier est un plaidoyer pro domo des enseignants d'Evora, au moment où les Réguliers du Tiers Ordre franciscain ont voulu créer un collège dans cette ville ${ }^{\left({ }^{80}\right)}$. Le second est une requête

p. 307.

( ${ }^{(4)}$ Annales d'Espagne et de Portugal, Amsterdam, 1741, t. III,

${ }^{\left({ }^{s 5}\right)}$ A.D.E. CXXVII/2-13, n० 2494, du 20 janvier 1773.

(*) A.D.E. CXXVII/2-13 n० 2501-2505, des 3, 8 et 15 mars 1775.

( $\left.{ }^{87}\right)$ A.D.E. CLXIV/2-33.

( ${ }^{\infty 8)}$ A.D.E. CXXVII/2-13, n० 2497, du 18 janvier 1775 et $n^{\circ} 2499$ du 21 février 1775 .

(") La civilisation de l'Europe des Lumières, Paris, 1971, p. 147.

${ }^{(\infty)}$ Boletim da Bibliografia portuguesa, vol. 2, fasc 5-5, 1881. L'auteur en serait d'ailleurs Bento Farinha, d'après A. A. Andrade. 
adressée par D. Manuel do Cenáculo, lui-même au souverain. L'évêque de Beja déclare que, dans son diocèse, on trouve nombre de jeunes gens ayant un bon niveau d'instruction et ce, grâce aux écoles que le Roi a fondées $\left({ }^{61}\right)$. Et, de fait, le collège de Beja, pour reprendre cet exemple, semble avoir pleinement réussi: les registres d'immatriculation nous montrent une population scolaire de l'ordre de 220 élèves, sur les trois années $1774-1776\left({ }^{62}\right)$. Mais, nous ne saurions étendre à tous les chefs lieux de comarcas l'exemple de la ville épiscopale du président de la commission! En effet, nombre de comarcas n'ont pas eu les enseignants prévus; si de petites villes, à cause de leur fonction, sont dotées de professeurs, des agglomérations plus importantes par contre n'en ont pas. Ces reproches, dressés par António Alberto Banha de Andrade, sont parfaitement justifiés ( ${ }^{63}$ ). Mais, les membres de la Real Mesa Censória ont quelques excuses; réaliser ce qu'ils ont pu faire, et ce, en quelques années, dans des conditions aussi difficiles, est déjà un succès.

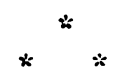

Dépassant le simple problème des réalisations, de leurs insuffisances ou de leur mauvaise répartition, nous devons plutôt nous attacher au principe qui a inspiré cette réforme. Avec celle-ci, nous avons un exemple type des Luzes, de ces Lumières méditérranéennes, avides de réalisations et pour lesquelles tout se tient. C'est le thème sous-jacent dans le texte de Domingos Vandelli: formez les gens de l'Alentejo grâce à des écoles et l'agriculture y sera aussi prospère que dans le nord du pays $\left({ }^{64}\right)$. L'oeuvre amorcée par Pombal et la Real Mesa Censória n'a pas été totalement abandonnée à la chute du grand ministre. D. Maria I a exigé la présence à leur poste des professeurs nommés; elle a aussi mis l'accent sur l'enseignement féminin. Mais, sous son règne, la réforme a été dépouillée de son esprit. Les écoles ont été de plus en plus abandonnées à des Congrégations: il est vrai que ces enseignants se

( ${ }^{61}$ A.D.E. CXXX/2-17. Copie d'une requête adressée au Roi, le 15 juin 1776 pour solliciter la permission d'ordonner de nouveaux prêtres.

(') A.D.E. CXXX/2-19.

("3) Art. cit.; Revista de História, 1977, p. 493.

(अ) Cf. note 20, p. 105. 
contentaient des $2 / 3$ du traitement d'un professeur royal $\left({ }^{85}\right)$. De plus, la création de nouvelles écoles est bien souvent abandonnée à l'initiative privée, donc à l'Eglise. Citons, entre autres, les réalisations de D. Manuel do Cenáculo dans la serra de Monchique $\left.{ }^{(68}\right)$, et de D. Gomes de Avelar, en Algarve $\left({ }^{67}\right)$ ou de $D$. Caetano Brandão, dans l'archidiocèse de Braga ${ }^{68}$ ). Que le nombre d'écoles n'ait cessé d'augmenter est un phénomène incontestable; mais c'en était fini de la grande idée de Pombal, un enseignement d'Etat, laïque. Il est toujours possible de souligner les limites de l'oeuvre accomplie, l'insuffisance des réalisations, ou bien de relever certaines erreurs ou maladresses. Toutefois, qu'il nous soit permis de rapprocher deux dates: 1773, début de l'application effective de la réforme, et 1777, chute de Pombal et ruine de son oeuvre. Pour une tâche de cette ampleur, il aurait fallu des décennies; quel que soit leur dévouement, D. Manuel do Cenáculo et ses collaborateurs, qui avaient en outre bien d'autres activités, n'ont disposé que de quelques années. Même si on n'en partage pas l'exagération, on ne peut s'empêcher de songer au jugement porté par Dumouriez au retour de son voyage au Portugal: "Il faudrait un siècle de gouvernement pareil à celui du comte d'Oeyras, pour mettre ce royaume dans l'état de force, de population, de cultivation, dont il est susceptible» $\left.{ }^{69}\right)$. Et, pour tout homme des Luzes, de Vernei $\left({ }^{70}\right)$ à Pombal et son entourage, la réforme des estudos menores était un des éléments-clefs d'une telle politique.

${ }^{\left({ }^{\circ 5}\right)}$ Dicionário de História de Portugal, II, pp. 48-53, art. ensino primário.

${ }^{\infty}$ ) A.D.E. CXXVIII/2-4. Note à son régisseur, du 6 janvier 1779.

( ${ }^{(7)}$ J. Leite de Vasconcelos, «A acção episcopal na formação e desenvolvimento das povoações portuguesas», Biblos, 7, 1931, pp. 97-111.

$\left({ }^{88}\right)$ B.A.C. $694 \mathrm{~V}$, ff. $85,92$.

("o) Ob. cit., p. 56.

$\left({ }^{70}\right)$ Verdadeiro método de estudar, Carta XVI. 
La réforme des «Estudos Menores»

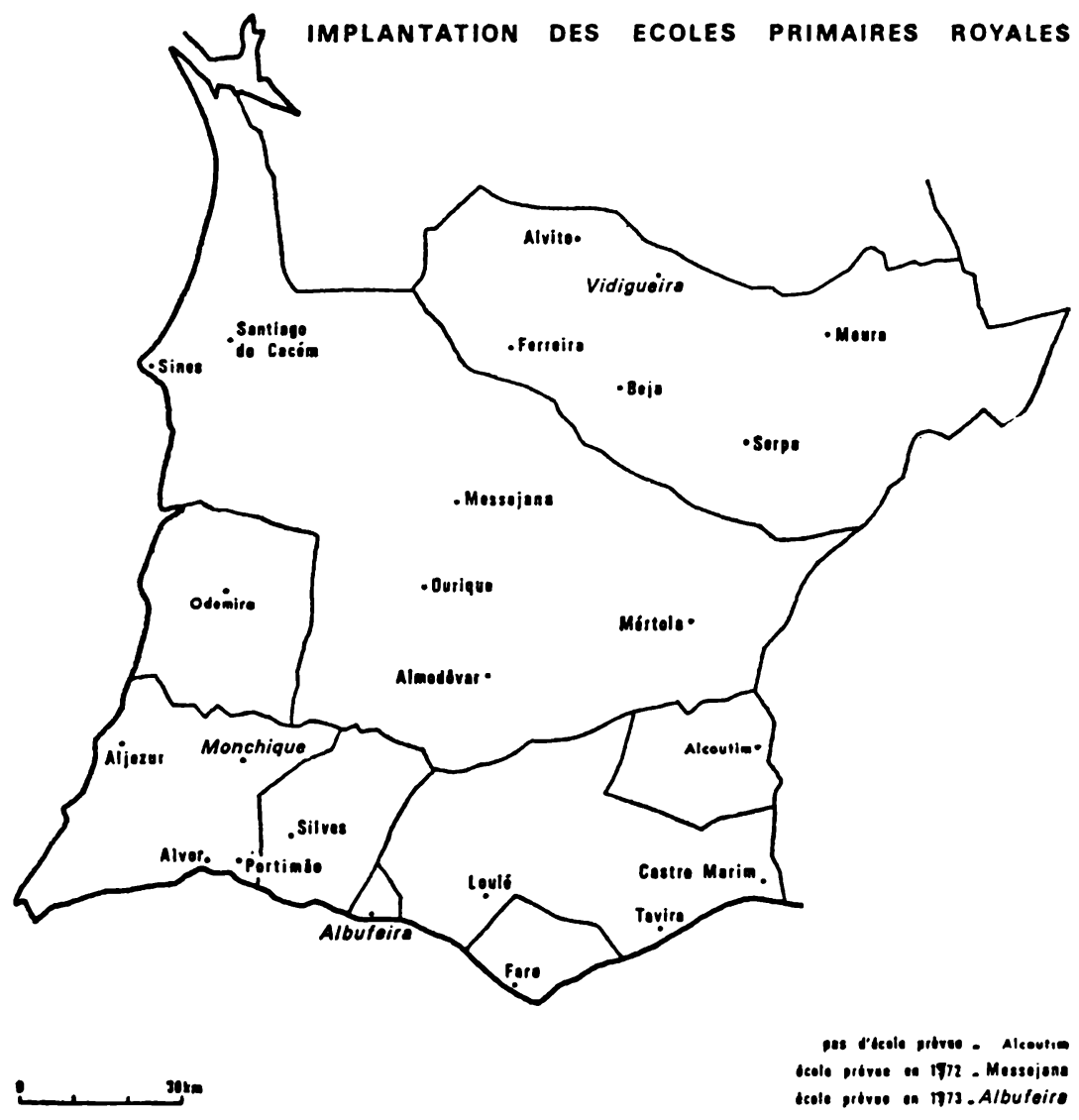

